\title{
Changes in the electronic structure of cerium due to variations in close packing
}

\author{
K. T. Moore, ${ }^{1, *}$ B. W. Chung, ${ }^{1}$ S. A. Morton, ${ }^{1}$ A. J. Schwartz, ${ }^{1}$ J. G. Tobin, ${ }^{1}$ S. Lazar, ${ }^{2}$ F. D. Tichelaar, ${ }^{2}$ H. W. Zandbergen, ${ }^{2}$ \\ P. Söderlind, ${ }^{3}$ and G. van der Laan ${ }^{4}$ \\ ${ }^{1}$ Chemistry and Materials Science Directorate, Lawrence Livermore National Laboratory, Livermore, California 94550, USA \\ ${ }^{2}$ National Center for High Resolution Electron Microscopy, Delft University of Technology, Rotterdamseweg 137, \\ 2628 AL Delft, The Netherlands \\ ${ }^{3}$ Department of Physics, Lawrence Livermore National Laboratory, Livermore, California 94550, USA \\ ${ }^{4}$ Synchrotron Radiation Source, Daresbury Laboratory, Warrington WA4 4AD, United Kingdom \\ (Received 24 September 2003; revised manuscript received 24 November 2003; published 14 May 2004)
}

\begin{abstract}
Here we use electron energy-loss spectroscopy in a monochromated transmission electron microscope with $100 \mathrm{meV}$ energy resolution and $2 \AA$ spatial resolution to show that the electronic structure of face centered cubic $\gamma$ and double hexagonal close-packed $\beta$ cerium are considerably different, contrary to previous assumptions in literature. These results are supported by synchrotron-radiation-based $\mathrm{x}$-ray absorption, multielectronic atomic spectral simulations, and local density approximation calculations, illustrating that changes in $\{111\}$ stacking sequences can drive substantial electronic changes in close-packed phases of cerium that have a similar atomic volume.
\end{abstract}

DOI: 10.1103/PhysRevB.69.193104

PACS number(s): 71.10.-w, 71.15.Mb, 71.20.Eh, 71.27.+a

Metals whose ground state lie at the transition between magnetic and superconductive behavior exhibit crystallographic phase instability. ${ }^{1-3}$ Near this transition, small changes in temperature, pressure, and chemistry can cause transformations to occur, often allowing multiple crystal phases to coexist in metastable equilibrium. Therefore, acquiring single-phase samples of metals at or near this transition, such as manganese (four phases), cerium (four phases), and plutonium (six phases), is uncertain, rendering spectroscopic techniques with low spatial resolution questionable. ${ }^{4}$ Producing large single crystals of these metals is even more difficult, and for many of them has yet to be accomplished. Rather than struggling to generate single-phase or singlecrystal samples of these complex metals, one may take the route of using an experimental technique that probes small portions of a sample, circumventing crystal growth problems. 5 Cerium, which is an archetypal correlated $f$-electron metal that exhibits notorious phase instability, has generated decades of experimental frustration and uncertainty, and is a benchmark metal with which to experiment with a small probe.

At ambient pressure, Ce metal exhibits four allotropic phases between absolute zero and its melting temperature at $1071 \mathrm{~K}: \alpha, \beta, \gamma$, and $\delta$. There are large hystereses between the transformations of $\alpha, \beta$, and $\gamma$, causing phase boundaries to be kinetic approximations and mixtures of two or even three phases to metastably persist in the thermodynamically single-phase fields. ${ }^{7,8}$ When fcc $\gamma$-Ce transforms to fcc $\alpha$-Ce upon cooling, it undergoes an isostructural volume collapse of $17 \%$. Several interpretations have been made as to why this collapse occurs, such as the promotion of the single localized $4 f$ electron, ${ }^{9}$ Kondo volume collapse, ${ }^{10}$ and a metalto-insulator Mott transition. ${ }^{11}$ However, this issue has yet to be resolved, and there is even new evidence showing a combination of effects. ${ }^{12}$

While there have been copious investigations of the $\alpha$ $\rightarrow \gamma$ transformation, the $\beta$ phase has been to a great degree ignored. This is because it has long been assumed that fcc $\gamma$-Ce and dhcp $\beta$-Ce have an almost identical electronic structure due to the fact both are close packed with a coordination number of 12 , have a similar atomic volume, and contain one $f$ electron that is completely, or close to completely, localized. ${ }^{1,13}$ At the same time, the coexistence of $\beta$ and $\gamma$ in cerium samples is pervasive and has plagued spectroscopic and structural experiments. Here, we overcome this problem by using electron energy-loss spectroscopy (EELS) in a monochromated transmission electron microscope (TEM), and present EELS spectra of $\gamma$ - and $\beta$-Ce, as evidenced by single-crystal diffraction patterns. Using EELS, synchrotron-radiation-based x-ray absorption (XAS), multielectronic spectral simulations with an atomic model, and local density approximation (LDA) calculations based on density-functional theory with the general gradient approximation, we show that there is in fact a significant difference in electronic structure between the fcc $\gamma$ phase and the dhcp $\beta$ phase of $\mathrm{Ce}$.

EELS spectra were acquired from Ce metal samples of 99.9\% purity. Samples were thinned to electron transparency in an ion mill, then vacuum transferred to the monochromated FEI Tecnai-200 kV field-emission-gun TEM to minimize oxidation. The monochromated electron source of the TEM [upper-right inset in Fig. 1(a)] enables $100 \mathrm{meV}$ energy resolution, which is about 10 times better than current TEM's at $1 \mathrm{eV}$. This increased energy resolution is imperative for $\mathrm{Ce}$ in order to resolve the fine structure of the $N_{4,5}(4 d \rightarrow 4 f)$ and $M_{4,5}(3 d \rightarrow 4 f)$ peaks. All spectra were reproduced numerous times using a sample thickness of approximately 0.5 inelastic mean free path $(40 \mathrm{~nm})$, as calculated by the zeroloss and plasmon peaks. Background removal was performed using a power-law extrapolation and various window placements to ensure correct and consistent background removal. Multiple spectra were recorded, aligned, and summed to optimize the signal-to-noise ratio and to maintain optimal energy resolution.

In the microscope, the $\beta$ and $\gamma$ phases could readily be identified in imaging mode by diffraction contrast, then verified via single-crystal diffraction patterns. The $\beta$ phase was either present initially at room temperature with $\gamma$ as a two- 

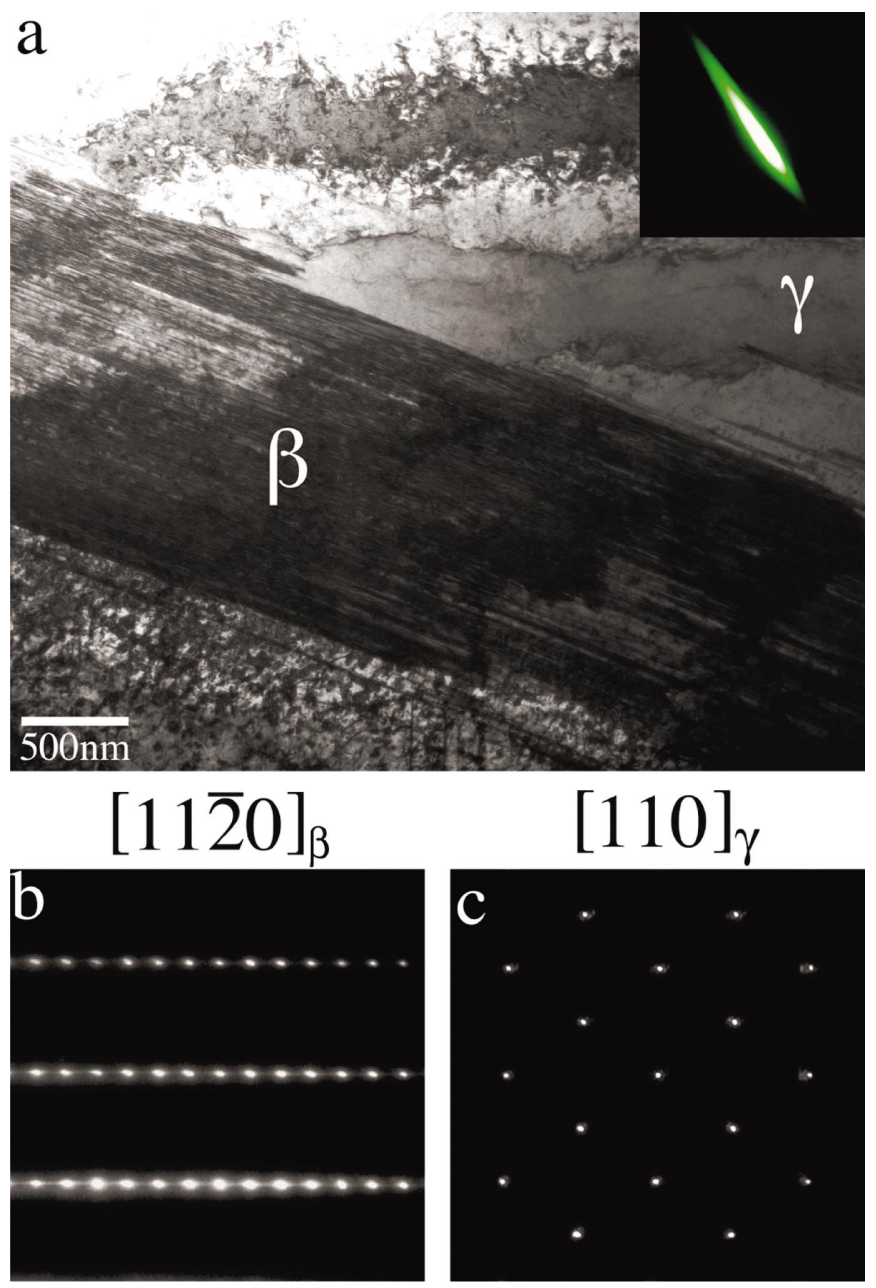

FIG. 1. (Color) (a) A bright-field TEM image of a two-phase mixture of $\beta$ - and $\gamma$-Ce near a $[11 \overline{2} 0]_{\beta} \|[110]_{\gamma}$ zone axis. (b) A [11 $\overline{2} 0]$ diffraction pattern from $\beta$ and (c) a [110] diffraction pattern from $\gamma$. In fcc, the $\{111\}$ planes repeat $\cdots A B C \cdots$, while in dhcp they repeat $\cdots A B A C \cdots$, meaning the $\operatorname{dhcp} \beta$ structure consists of alternating cubic and hexagonal layers. While single-phase samples of $\beta$-Ce can be produced (Ref. 30), for a number of reasons it is exceedingly difficult to prepare the samples for analysis by XAS. For instance, when a bulk sample of $\beta$ cerium is filed, sanded, or sputter cleaned and annealed, the surface transforms to $\gamma$-Ce. This leaves the option of growing a thin film of $\beta$-Ce. However, attempts at this (Refs. 13, 31) have proved ambiguous with respect to which phase is actually being examined. The ambiguity arises due to the fact that low-energy electron diffraction yields a hexagonal pattern that could be either (111) fcc- $\gamma$ or (0001) dhcp- $\beta$. The monochromated TEM removes this uncertainty. The upper-right inset in (a) shows the monochomated electron beam prior to insertion of the energy-selecting slit. The beam is elongated as a function of energy, allowing the removal of electrons with large positive or negative energy deviations.

phase mixture, or was formed by repeatedly cooling a $\gamma$-Ce sample in situ with a liquid-He holder. The process of phase identification is illustrated in Fig. 1, where (a) is a brightfield TEM image of a two-phase mixture of $\beta$ - and $\gamma$-Ce, and (b) and (c) are single-crystal diffraction patterns of $\beta$ and $\gamma$, respectively. The electron beam can be focused so that EELS spectra can be recorded from an area with a diameter of $2 \AA$. Upon examination of the scale bar in Fig. 1(a), it is obvious that the problem of sampling multiple phases when recording spectra from crystallographically unstable metals is alleviated.

X-ray absorption spectra were acquired at Beamline 7.0 at the Advanced Light Source ${ }^{14}$ from Ce thin films deposited on a $\mathrm{Cu}$ substrate at $170 \mathrm{~K}$. A base vacuum of $10^{-11}$ Torr was maintained to ensure minimal surface oxidation, except during deposition, when the pressure elevated to $10^{-10}$ Torr. A wide-scan photoemission spectra taken at $h v=1250 \mathrm{eV}$ showed no detectable $\mathrm{O} 1 s, 2 p$, or $2 s$ peak above the noise, indicate minimal oxygen on the sample. Since the oxygen cross section is low and the mean free path is large at $h v$ $=1250 \mathrm{eV}$, we also performed a higher resolution scan using $h v=127 \mathrm{eV}$ and found the $\mathrm{O} 2 p$ edge was almost nonexistent. It should be noted that this is operating on resonance. However, studies of Pu (Ref. 15) have shown that even on resonance the sensitivity of the $\mathrm{O} 2 p$ peak to slight amount of oxidation remains high.

Theoretical X-ray absorption spectra were calculated in intermediate coupling using Cowan's relativistic HartreeFock code ${ }^{16}$ taking into account Coulomb, exchange, and spin- orbit interaction on an equal footing. Resonant Auger decay to the photoemission continuum states was included using the $t$-matrix approach, ${ }^{17}$ which gives the lifetime of the individual multiplet lines and hence their broadening. Therefore, no artificial broadening needed to be applied.

The $N_{4,5}(4 d \rightarrow 4 f)$ and $M_{4,5}(3 d \rightarrow 4 f)$ edges of Ce are shown in Fig. 2, where the upper spectrum is XAS, the middle is EELS, and the lower is a multielectronic atomic calculation. ${ }^{17-19}$ There are two important points to notice here. First, the extremely close match between the multielectronic atomic calculations and the EELS and XAS spectra, including the fine structure of both the $N_{4,5}$ and $M_{4,5}$ transitions, illustrate that the spectral simulations have indeed captured the fundamental underlying physics. Second, the close comparison between the XAS and EELS spectra demonstrate that the EELS transitions are certainly operating in the dipole limit, as observed previously. ${ }^{5}$ This affords confidence in interpretation of the EELS spectra.

The rare-earth metals exhibit the same $N_{4,5}(4 d \rightarrow 4 f)$ spectral shape, where a pre-peak structure emanates at lower photon energies followed by a broad continuum peak at higher photon energies. ${ }^{20}$ This behavior has been explained previously by Dehmer, ${ }^{21}$ Starace, ${ }^{22}$ and Sugar, ${ }^{23}$ where the Coulomb and exchange interactions between the partially occupied $4 d$ and $4 f$ final state levels drive the splitting of these angular-momentum-coupled states, producing an energy splitting on the scale of $20 \mathrm{eV}$. The prepeak structure remains as individual peaks but at higher photon energies, where the outgoing electron can actually escape, coupling to the continuum states generates a large, broad peak, that is referred to as the giant resonance. ${ }^{24}$ The prepeaks are of atomic character, and thus, in $\mathrm{Ce}$ are rather insensitive to the phase of the metal. Only in $\alpha$-Ce is a slight broadening of the pre-peaks observed due to $d-f$ hybridization. ${ }^{25}$ Therefore, it is within the giant resonance that one must look for electronic changes between different phases of $\mathrm{Ce}$. 


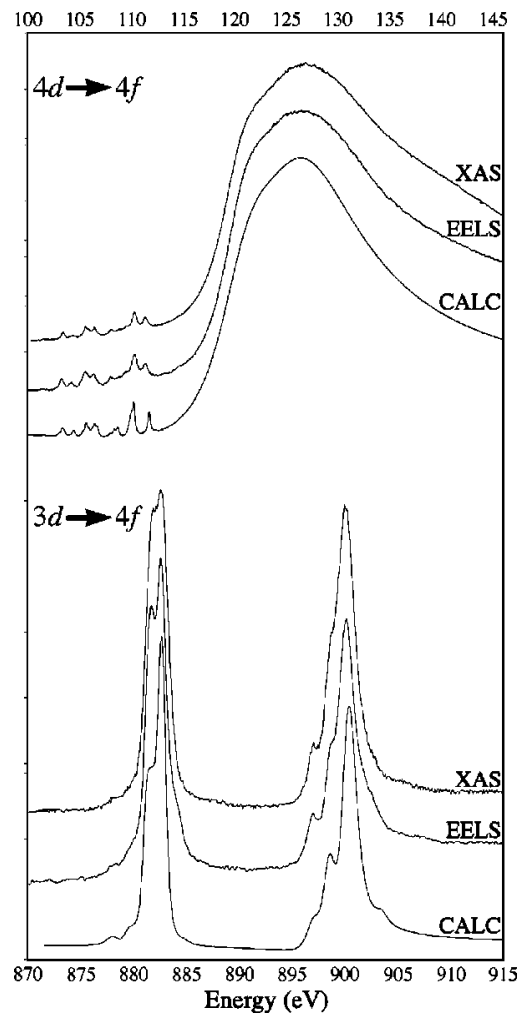

FIG. 2. A series of spectra showing both the $N_{4,5}(4 d \rightarrow 4 f)$ and $M_{4,5}(3 d \rightarrow 4 f)$ transitions for cerium. The ability of the multielectronic atomic calculations to replicate the XAS and EELS spectra so closely demonstrates the strongly atomic nature of $f$ electrons in cerium metal. Notice that the spectra from XAS and EELS from a monochromated TEM are essentially identical in both resolution and form. The TEM's ability to form a $2 \AA$ probe means that spectral investigations of highly site-specific regions of a sample, such as interfaces, dislocations, grain boundaries, etc., can be performed with very high energy resolution. The additional capability to record images and diffraction patterns is of great importance for lanthanide and actinide metals where a mixture of multiple crystallographic phases is common.

When we overlay the $N_{4,5}(4 d \rightarrow 4 f)$ EELS edges for $\beta$ and $\gamma$-Ce in Fig. 3(a), we find that while the prepeaks are unchanged, the giant resonance of $\beta$ is more pointed and about $2 \mathrm{eV}$ narrower. This is a surprisingly large difference between the two phases. While we would like to simulate the spectral differences, calculations that take into account the initial state, final state, and crystal structure have yet to reach the capability to describe transitions into $f$ states. The atomic calculations used here take into account multiplet structure, core-hole effects, and autoionization, but they are insensitive to crystal structure. This means that the structural differences between $\beta$ and $\gamma$ Ce due to stacking sequence of the closepacked $\{111\}$ planes cannot be accounted for with such calculations. Therefore, we calculated the $s, p, d$, and $f$ projected density of states (DOS) for both fcc $\gamma$ - and dhcp $\beta$-Ce using DFT. ${ }^{26}$ In these projected DOS in Figs. 3(b) and 3(c), it can be seen that the $f$ spectral weight is higher in peak A for $\gamma$-Ce, while it is higher in peak B for $\beta$-Ce. Also, the overall $f$ spectral weight is centered at a slightly lower energy in $\beta$. The calculated DOS also indicate that valence-band photo-
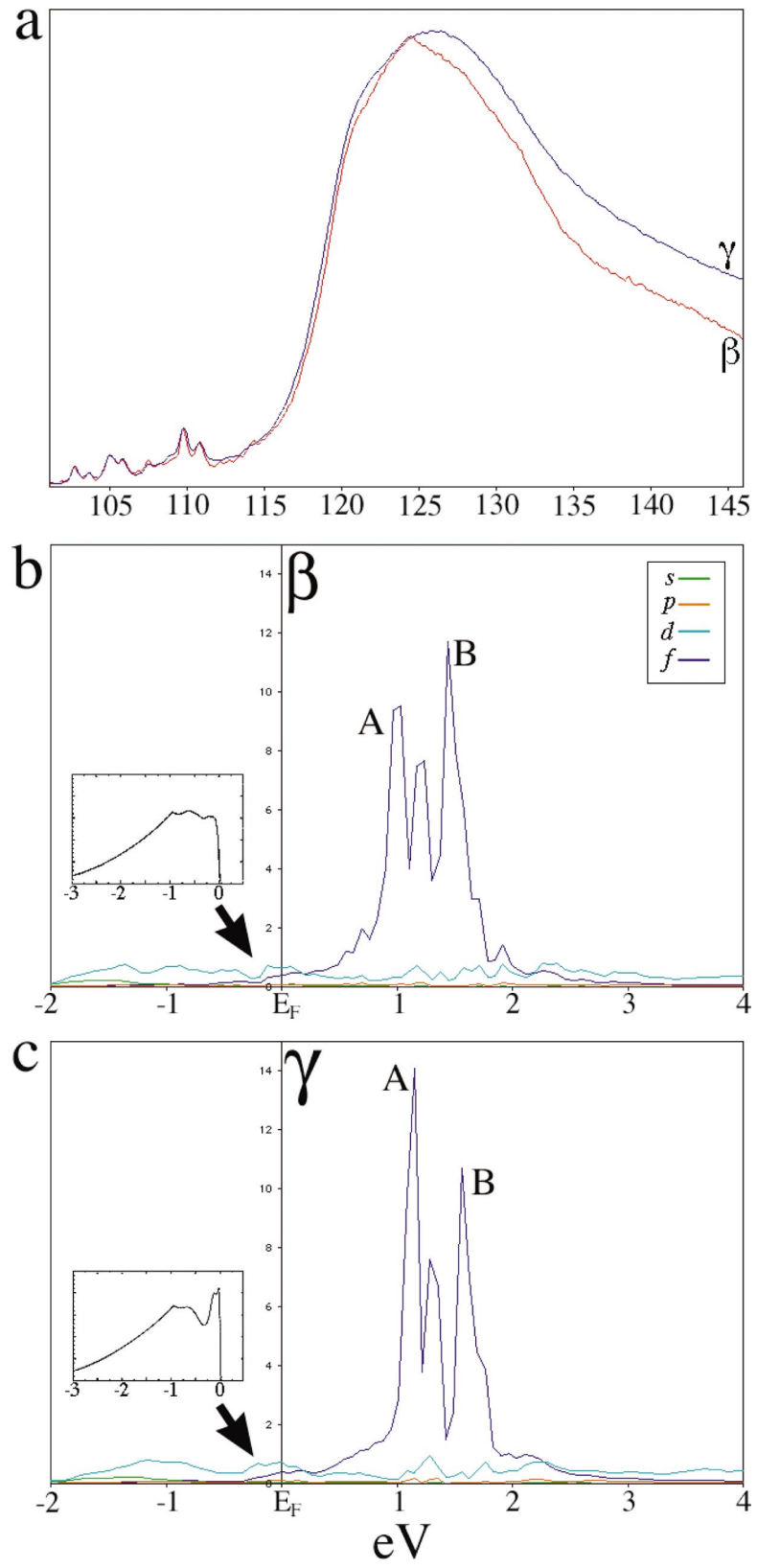

FIG. 3. (Color) (a) The $N_{4,5}(4 d \rightarrow 4 f)$ EELS edge for both $\beta$ and $\gamma$-Ce. The giant resonance of the $\beta$ spectra is approximately 2 $\mathrm{eV}$ narrower and more pointed than the $\gamma$ spectra, which is a surprisingly large difference between two close-packed metals with a similar atomic volume. (b) and (c) The calculated $s, p, d$, and $f$ projected density of states (DOS) for (b) $\beta$ and (c) $\gamma$. A coarse mesh was used in the calculation so that differences between the spectra could be more easily visualized. The differences in the $f$ projected DOS between $\gamma$ and $\beta$ are evident by examining peaks A and B, which shift in weight between the two phases. Notice that the occupied DOS below the Fermi level [arrowed in (b) and (c)] are different for $\gamma$ and $\beta$, indicating that valence-band photoemission spectra should be considerably different for the two phases.

emission spectra of the occupied DOS of $\beta$ and $\gamma$ should be different. This can be seen by examining the arrowed inset in both Figs. 3(b) and 3(c). Here, the dominant peak at the Fermi level and the shoulder at approximately $200 \mathrm{meV}$ are 
markedly smaller in the $\beta$ DOS calculations, indicating a higher DOS near the Fermi energy for $\gamma$-Ce relative to $\beta$-Ce.

The salient point of these results is that our experimental and calculated spectra suggest $\beta$ - and $\gamma$-Ce have different $f$ electronic structure, contrary to assumptions in previous literature. Here, simple changes in stacking sequence seem to drive considerable modifications in the $f$ electronic structure. For the lanthanides, the observed structural sequence hcp $\rightarrow$ samarium-type $\rightarrow$ dhcp $\rightarrow$ fcc has been explained in terms of $d$-band occupancy $\left(N_{d}\right)$ by Duthie and Pettifor, ${ }^{27}$ where an increase in $N_{d}$ from 1.5 to 2.7 fuels the structural change. The dhcp structure is stabilized by an occupation of $d$ electrons between 2.2 to $2.5 N_{d}$. However, here we see that there are considerable changes in the $f$ electronic structure of $\mathrm{Ce}$ due to changes in $\{111\}$ stacking sequence and, therefore, that the electronic structure of "localized" or " $\gamma$-like" phases in Ce is not solely due to valence $d$ electrons. In condensed matter, the outer electron wave functions overlap, and if their energy and symmetry is appropriate, hybridization will arise. The hybridization strength between the $4 f$ and $s, p$, and $d$ band states in the lanthanides depends not only on the energy and symmetry of the available states, but on the arrangement and number of the neighboring atoms, ${ }^{28}$

*Electronic address: moore78@1lnl.gov

${ }^{1}$ D. C. Koskenmaki, K. A. Gschneidner, Jr., in Handbook on the Physics and Chemistry of Rare Earths, edited by K. A. Gschneidner, Jr. and L. R Eyring (North-Holland, Amsterdam, 1978), Chap. 4.

${ }^{2}$ J. L. Smith and E. A. Kmetko, J. Less-Common Met. 90, 83 (1983).

${ }^{3}$ S. Y. Savrasov, G. Kotliar, and E. Abrahams, Nature (London) 410, 793 (2001).

${ }^{4}$ D. Ottewell, E. A. Stewardson, and J. E. Wislon, J. Phys. B 6, 2184 (1973).

${ }^{5}$ K. T. Moore, M. A. Wall, A. J. Schwartz, B. W. Chung, D. K. Shuh, R. K. Schulze, and J. G. Tobin, Phys. Rev. Lett. 90, 196404 (2003).

${ }^{6}$ J. Wong, M. Krisch, D. L. Farber, F. Occelli, A. J. Schwartz, T.-C. Chiang, M. Wall, C. Boro, and R. Xu, Science 301, 1078 (2003).

${ }^{7}$ C. J. McHargue and H. L. Yakel, Jr., Acta Metall. 8, 637 (1960).

${ }^{8}$ M. S. Rashid and C. J. Altstetter, Trans. Metall. Soc. AIME 236, 1649 (1966).

${ }^{9}$ A. W. Lawson and T. Y. Tang, Phys. Rev. 76, 301 (1949).

${ }^{10}$ J. W. Allen and R. M. Martin, Phys. Rev. Lett. 49, 1106 (1982).

${ }^{11}$ B. Johansson, Philos. Mag. 30, 469 (1974).

${ }^{12}$ Y. Held, A. K. McMahan, and R. T. Scalettar, Phys. Rev. Lett. 87, 276404 (2001).

${ }^{13}$ E. Weschke, A. Höhr, G. Kaindl, S. L. Molodtsov, S. Danzenbächer, M. Richter, and C. Laubschat, Phys. Rev. B 58, 3682 (1998)

${ }^{14}$ S. A. Morton, G. D. Waddill, S. Kim, Ivan K. Schuller, S. A. Chambers, and J. G. Tobin, Surf. Sci. 513, L451 (2002).

${ }^{15}$ J. G. Tobin, B. W. Chung, R. K. Schulze, J. Terry, J. D. Farr, D. K. Shuh, K. Heinzelman, E. Rotenberg, G. D. Waddill, and G. van der Laan, Phys. Rev. B 68, 115109 (2003).

${ }^{16}$ R. D. Cowan, The Theory of Atomic Structure and Spectra (University of California Press, Berkeley, 1981). again indicating the importance of the crystal structure.

The unoccupied density of states must also be considered in the lanthanides due to the strong mixing of the states. For example, it has been shown by Temmerman, Szotek, and Winter ${ }^{29}$ that the unoccupied $4 f$ bands in Pr metal hybridized strongly with the $s, p$, and $d$ valence bands. This means that the first empty $4 f$ level is close enough to the Fermi level that it mixes with the $(s p d)$ valence electrons, altering the electronic structure of the metal. This behavior is again affected by crystal structure. Therefore, both the occupied and unoccupied density of states of $\mathrm{Ce}$ is affected by changes in crystal structure. Other $f$-electron metals that exhibit multiple phases near large volume changes due to localizeditinerant transitions, such as plutonium with temperature and americium with pressure, may also subject to these results.

Special thanks to Gil Gallegos and Paul Sandoval for cerium samples. This work was performed under the auspices of the U.S. DOE by Lawrence Livermore National Laboratory under Contract No. W-7405-Eng-48, with support from Lawrence Berkeley National Laboratory (Contract No. DEAC03-76SF00098). The Advanced Light Source and the Spectromicroscopy Facility (Beamline 7.0) have been built and operated under funding from the Office of Basic Energy Science at Department of Energy.

${ }^{17}$ G. van der Laan, E. Arenholz, Z. Hu, A. Bauer, E. Weschke, Ch. Schüssler-Langeheine, E. Navas, A. Mühlig, G. Kaindl, J. B. Goedkoop, and N. B. Brookes, Phys. Rev. B 59, 8835 (1999).

${ }^{18}$ B. T. Thole, G. van der Laan, J. C. Fuggle, G. A. Sawatzky, R. C. Karnatak, and J.-M. Esteva, Phys. Rev. B 32, 5107 (1985).

${ }^{19}$ G. van der Laan, B. T. Thole, G. A. Sawatzky, J. C. Fuggle, and R. Karnatak, J. Phys. C 19, 817 (1986).

${ }^{20}$ W. D. Schneider, in Electronic Structure of Solids: Photoemission Spectra and Related Data, edited by A. Goldmann and E. Koch, Landolt-Bornstein Numerical Data and Functional Relationships in Science and Technology, Group III (Springer-Verlag, Berlin, 1994), Vol. 23a, Chap. 2.5.

${ }^{21}$ J. L. Dehmer, A. F. Starace, U. Fano, J. Sugar, and J. W. Cooper, Phys. Rev. Lett. 26, 1521 (1971).

${ }^{22}$ A. F. Starace, Phys. Rev. B 5, 1773 (1972).

${ }^{23}$ J. Sugar, Phys. Rev. B 5, 1785 (1972).

${ }^{24}$ S. R. Mishra, T. R. Cummins, G. D. Waddill, W. J. Gammon, G. van der Laan, K. W. Goodman, and J. G. Tobin, Phys. Rev. Lett. 81, 1306 (1998).

${ }^{25}$ D. Wieliczka, J. H. Weaver, D. W. Lynch, and C. G. Olson, Phys. Rev. B 26, 7056 (1982).

${ }^{26}$ P. Söderlind, O. Eriksson, B. Johansson, and J. M. Wills, Phys. Rev. B 52, 13169 (1995).

${ }^{27}$ J. C. Duthie and D. G. Pettifor, Phys. Rev. Lett. 38, 564 (1977).

${ }^{28}$ Y. Baer, W. D. Schneider, in Handbook on the Physics and Chemistry of Rare Earths, edited by K. A. Gschneidner, Jr., L. R. Eyring, and S. Hüfner (North-Holland, Amsterdam, 1987), Vol. 10, Chap. 62, p. 16.

${ }^{29}$ W. M. Temmerman, Z. Szotek, and W. Winter, Phys. Rev. B 47, 1184 (1993).

${ }^{30}$ D. C. Koskimaki, K. A. Gschneidner, Jr., and N. T. Panousis, J. Cryst. Growth 22, 225 (1974).

${ }^{31}$ Y. Tanaka, M. Kamel, and Y. Gotoh, J. Cryst. Growth 169, 299 (1996). 